\title{
Human Platelets Modulate Edema Formation in Isolated Rabbit Lungs
}

\author{
John E. Heffner, James A. Cook, and Perry V. Halushka \\ Department of Medicine, Department of Physiology, and the Department of Cell and Molecular Pharmacology and Experimental \\ Therapeutics, Medical University of South Carolina, Charleston, South Carolina 29425
}

\begin{abstract}
The role of platelet glucose-6-phosphate dehydrogenase (G-6PD) in mediating the effects of human platelets on oxidant-induced edema in the isolated perfused rabbit lung was investigated using dehydroepiandrosterone, a specific steroidal inhibitor of G-6-PD. Xanthine oxidase $(0.003$ and $0.012 \mathrm{U} / \mathrm{ml})$ caused lung edema that was attenuated by coinfusion of washed human platelets. Platelets that were incubated with DEA to inhibit G-6-PD activity augmented xanthine oxidase-induced lung edema and pulmonary hypertension at both doses of xanthine oxidase. Infusion of papavarine to maintain stable pulmonary artery (PA) pressures, incubation of G-6-PD-inhibited platelets with acetylsalicylate, or infusion of a thromboxane-prostaglandin endoperoxide receptor site antagonist, SQ 29548, into the lung perfusate prevented augmentation of lung edema and the PA pressor response by G-6-PD-inhibited platelets. It was concluded that antioxidant-intact platelets attenuate oxidant-induced lung edema by preventing increased membrane permeability, and that G-6-PD-inhibited platelets augment lung edema through hydrostatic mechanisms mediated by release of platelet cyclooxygenase products.
\end{abstract}

\section{Introduction}

Considerable clinical and experimental data indicate that platelets are important regulators of vascular permeability. Platelets maintain vascular integrity as demonstrated by capillary fragility, purpura, and petechiae that are common clinical features of thrombocytopenia $(1,2)$. Furthermore, organ tissue edema is enhanced after platelet depletion in whole animals (3, 4) and isolated perfused organs (5-7) at basal states and after exposure to injurious substances, such as $\alpha$-naphthylthiourea (8). Mechanisms underlying platelet protection of vascular permeability, however, remain unclear.

Platelets also have potential mechanisms of cytotoxicity that may contribute to increased vascular permeability in certain conditions (9). Thrombin-stimulated platelets release vasoactive substances that loosen and injure cultured endothelial cell monolayers (10), stimulation of platelets by antigen-anti-

This paper was presented in part at the Annual Meeting of the American Thoracic Society, New Orleans, LA, 8 May 1988 and was published in abstract form in Am. Rev. Respir. Dis. 137:A77. [Abstr.]

Address reprint requests to Dr. John E. Heffner, Associate Professor, Division of Pulmonary and Critical Care Medicine, Medical University of South Carolina, Charleston, SC 29425.

Received for publication 7 September 1988 and in revised form 24 February 1989.

J. Clin. Invest.

(C) The American Society for Clinical Investigation, Inc.

0021-9738/89/09/0757/08 \$2.00

Volume 84, September 1989, 757-764 body complexes cause vascular injury and inflammation in rabbits (11), and thrombocytopenia attenuates vascular injury after complement activation by cobra venom factor (12). Because activated platelets release potent vasoconstrictors, such as 5-hydroxytryptamine, histamine, adenine nucleotides, prostaglandin endoperoxides, and $\operatorname{TXA}_{2}(13,14)$, platelets may further indirectly enhance membrane permeability edema in conditions such as edematous lung injury by causing pulmonary hypertension and increased intracapillary hydrostatic pressure (15).

Whether platelets are beneficial or deleterious to membrane permeability in the pulmonary microvasculature is a particularly important question. Biopsy and autopsy specimens from patients with the adult respiratory distress syndrome (ARDS) ${ }^{1}$ demonstrate that platelets sequester in regions of lung injury $(16,17)$. Elucidation of the role of platelets in the pathogenesis of ARDS, however, has been lacking (18). We have previously demonstrated that human platelets can attenuate edema in the isolated perfused rabbit lung infused with xanthine oxidase (an enzyme that simulates neutrophil release of continuous low levels of several toxic oxygen metabolites), and that the protective effect is mediated through the platelet glutathione redox cycle (19). These findings suggest that platelets can reduce extracellular toxic oxygen metabolites ex vivo and that platelet antioxidant activity may be an important determinant of platelet interactions with vascular endothelium.

$3 \beta$-Hydroxy-5-androsten-17-one (dehydroepiandrosterone) is a selective steroidal inhibitor of the hexose monophosphate shunt enzyme glucose-6-phosphate dehydrogenase (G-6-PD) $(20,21)$. G-6-PD is an important intracellular antioxidant defense mechanism in that it supplies NADPH for normal functioning of the glutathione redox cycle $(22,23)$ and is stimulated after exposure of platelets to extracellular $\mathrm{H}_{2} \mathrm{O}_{2}$ (24). We reasoned that normal platelets in the presence of xanthine oxidase may attenuate oxidant-induced edema by reducing toxic oxygen metabolites, and that G-6-PD-inhibited platelets may become oxidant targets themselves, thereby enhancing lung injury through the release of cytotoxic or vasoconstrictive substances. In this study, the effect of dehydroepiandrosterone-treated human platelets on xanthine oxidase-induced oxidant lung edema in the isolated perfused rabbit lung was determined.

\section{Methods}

Materials. Male and female New Zealand White rabbits weighing between 2 and $2.5 \mathrm{~kg}$ were used in all experiments. Animals were housed individually in stainless steel cages in a temperature-controlled environment. $\left[5,6,8,-9,11,12,14,15^{3} \mathrm{H}\right] \mathrm{TXB}_{2}$ was obtained from New En-

1. Abbreviations used in this paper: ARDS, adult respiratory distress syndrome; G-6-PD, glucose-6-phosphate dehydrogenase; i, immunoreactive; $L V$, left ventricular; PA, pulmonary artery. 
gland Nuclear (Boston, MA). Authenic $\mathrm{TXB}_{2}$ for the immunoreactive (i) $\mathrm{TXB}_{2}$ assay was purchased from the Upjohn Co. (Kalamazoo, MI). The antagonist of the $\mathrm{TXA}_{2}$-prostaglandin endoperoxide receptor, [1S-[1a,2b(5Z),3b,4a]]-7-[3-[[2-[(phenylamino)-carbonyl]hydrazino]methyl]-7-oxabicyclo(2.2.1)hept-2-yl]-5-heptenoic acid (SQ 29548) was a kind gift of Martin Ogletree, Squibb Pharmaceuticals (Princeton, $\mathrm{NJ}$ ). Unless otherwise stated, all other chemicals were obtained from Sigma Chemical Co. (St Louis, MO).

Preparation of washed human platelets. $100 \mathrm{ml}$ venous blood was drawn using a two-syringe venipucture technique from healthy volunteers who were medication free for $14 \mathrm{~d}$. The syringes contained a citrate solution anticoagulant consisting of $0.085 \mathrm{M}$ trisodium citrate, $0.065 \mathrm{M}$ citric acid, and 2\% dextrose in a 1:6 dilution. Platelet-rich plasma (PRP) was prepared by centrifugation of the citrated blood at $275 \mathrm{~g}$ for $10 \mathrm{~min}$ at $24^{\circ} \mathrm{C}$. The PRP was centrifuged at $1,000 \mathrm{~g}$ for 15 min at $24^{\circ} \mathrm{C}$ to form a platelet pellet. The overlying platelet-poor plasma was removed with a plastic transfer pipette, and the pellet was resuspended in $\mathbf{4 0 ~} \mathrm{ml}$ of Krebs-Henseleit physiologic salt solution that was modified to exclude calcium. The suspension of washed platelets contained $1.20 \pm 0.07 \times 10^{10}$ cells.

In some experiments, platelets were incubated with agents to inhibit G-6-PD and cyclooxygenase enzyme before infusion into the isolated lung. To inhibit platelet G-6-PD, PRP was incubated with dehydroepiandrosterone $\left(10^{-6} \mathrm{M}\right)$ for $30 \mathrm{~min}$ at $24^{\circ} \mathrm{C}$ before preparing washed platelets. Platelet G-6-PD activity after incubation with dehydroepiandrosterone was determined by measuring the generation of NADPH by treated platelets and expressed as nanomoles of NADPH generated per minute by $10^{9}$ platelets (25). Glutathione content of platelets incubated with dehydroepiandrosterone was determined using Ellman's reagent and a modification of the glutathione-specific assay of Tietze (26). The final reaction mixture contained $200 \mu \mathrm{l}$ of sonicated washed platelets, $6 \mathrm{mM}$ NADPH, and $6 \mathrm{mM}$ dinitrobenzene in a $0.1 \mathrm{M}$ sodium phosphate buffer ( $\mathrm{pH} 8.0)$ containing $5 \mathrm{mM}$ EDTA. Glutathione concentration was expressed as nanomoles of glutathione per $10^{9}$ platelets.

To inhibit both platelet G-6-PD and cyclooxygenase enzyme, PRP was incubated with dehydroepiandrosterone $\left(10^{-6} \mathrm{M}\right)$ and acetylsalicylate $(100 \mu \mathrm{M})$ for $30 \mathrm{~min}$ at $24^{\circ} \mathrm{C}$ before centrifugation and resuspension in Krebs-Henseleit solution.

Preparation of isolated rabbit lungs. After anesthesia with intramuscular xylazine $(10 \mathrm{mg} / \mathrm{kg}$; Cutter Laboratory, Shawnee, KS) and intravenous ketamine (25-50 mg/kg; Parke-Davis \& Co., Detroit, MI), rabbit heart-lung preparations were removed and isolated. Briefly, the lungs were ventilated through a tracheotomy by a small animal ventilator (model 6700; Harvard Apparatus Co., S. Natick, MA) at 24 breaths/min with a tidal volume of $10-15 \mathrm{ml} / \mathrm{kg}$ body weight and $2 \mathrm{~cm}$ $\mathrm{H}_{2} \mathrm{O}$ positive end-expiratory pressure. Animals were ventilated with a mixture of $95 \% \mathrm{O}_{2}$ and $5 \% \mathrm{CO}_{2}$ that maintained perfusate $\mathrm{pH}$ and gas tensions at $\mathrm{pH}=7.38-7.46, \mathrm{PCO}_{2}=34-36 \mathrm{mmHg}$, and $\mathrm{PO}_{2}$ $=490-520 \mathrm{mmHg}$. After a midsternal thoracotomy, heparin (500 U) was injected into the right ventricle and the pulmonary artery was cannulated. A second cannula was inserted into the left ventricle (LV) and the heart-lung preparation was suspended in a humidification dome $\left(38^{\circ} \mathrm{C}\right)$ from a force-displacement transducer (type 4-327-0010; Grass Instruments, Quincy, MA). The lungs were perfused with a constant flow $(100 \mathrm{ml} / \mathrm{min}$ ) by a peristaltic pump (model 1203; Harvard Apparatus Co.) in a recirculating system containing Krebs-Henseleit physiologic salt solution and 3\% BSA. After the lungs were flushed with an initial $500 \mathrm{ml}$ of perfusate to remove residual blood cells and plasma, a recirculating system was established with $300 \mathrm{ml}$ of perfusate. Pulmonary artery (PA) pressure was measured throughout the experiments with a pressure transducer (Bell and Howell Co., Pasadena, CA) and recorded (recorder obtained from Grass Instruments).

Measurement of vascular injury in the isolated lung. Changes in lung weight were continuously measured by the force-displacement transducer and recorded on a polygraph (Grass Instruments). Increases in lung weight were attributed to lung edema. After $60 \mathrm{~min}$ of lung perfusion, experiments were terminated and the left lung was isolated and lavaged with $20 \mathrm{ml}$ of isotonic saline. The lung lavage specimens were assayed for albumin concentrations by the bromocresol green method (Technical Bulletin no. 631; Sigma Chemical Co.). Increases in airway albumin content were considered to reflect lung edema formation.

Measurements of perfusate platelet, erythrocyte, and neutrophil counts. 1-ml perfusate samples were drawn from the LV cannula after the lungs were flushed with $500 \mathrm{ml}$ of perfusate and anticoagulated with EDTA to determine perfusate counts of circulating cellular elements. Perfusate platelet counts were performed (Pacer Plus Counter; Coulter Electronics Inc., Hialeah, FL); platelet counts $<5,000$ cells $/ \mu 1$ were confirmed to be background counts by performing hemocytometer platelet counts. Erythrocyte and neutrophil counts in perfusate samples were determined by hemocytometer techniques.

Perfusate sample collection and measurement methods for iTX $B_{2}$ Perfusate samples $(3 \mathrm{ml})$ were drawn from the LV cannula at 15,25 , 45 , and $60 \mathrm{~min}$ of the experiments and collected in plastic tubes containing $0.1 \mathrm{ml}$ of indomethacin solution $(1 \mathrm{mg}$ indomethacin dissolved in $1 \mathrm{ml}$ of $0.1 \mathrm{M}$ sodium phosphate buffer, $\mathrm{pH} \mathrm{8.0)}$ ) for determination of iTXB ${ }_{2}$, the stable metabolite of $\mathrm{TXA}_{2}$. The tubes were centrifuged at $1,500 \mathrm{~g}$ and the supernatant was stored at $-20^{\circ} \mathrm{C}$ until assay. $\mathrm{TXX}_{2}$ levels were determined using previously described RIA methods (27). The iTXB ${ }_{2}$ antibody does not significantly cross-react with other prostaglandins $(<0.04 \%)$. The interassay variability of the $\mathrm{iTXB}_{2}$ assay is $10 \%$.

Infusion of purine and xanthine oxidase. Lungs were perfused for 15 min to establish a stable baseline lung weight and PA perfusion pressure. Purine ( $2 \mathrm{mM}$ ) was then instilled into the reservoir followed 5 min later (20 min of total perfusion) by xanthine oxidase. Experiments were performed using two concentrations of xanthine oxidase that were 0.003 or $0.012 \mathrm{U} / \mathrm{ml}$ perfusate. The lungs were perfused for an additional 40 min monitoring lung weight and PA pressure.

Infusion of platelets with purine and xanthine oxidase. After a 15-min stabilization period, $40 \mathrm{ml}$ of perfusate was withdrawn from the reservoir and purine $(2 \mathrm{mM})$ was instilled. After an additional 4 min of perfusion, $40 \mathrm{ml}$ of washed platelets $\left(1.20 \pm 0.07 \times 10^{10}\right.$ cells) was added to the reservoir. $1 \mathrm{~min}$ later ( $20 \mathrm{~min}$ of total lung perfusion), either 0.003 or 0.012 units $/ \mathrm{ml}$ of xanthine oxidase was instilled. The lungs were observed for an additional $40 \mathrm{~min}$.

Infusion of G-6-PD- and cyclooxygenase-inhibited platelets with purine and xanthine oxidase. Protocols using platelets preincubated with dehydroepiandrosterone alone to inhibit G-6-PD or preincubated with dehydroepiandrosterone plus acetylsalicylate to inhibit both G-6PD and cyclooxygenase enzyme were performed at both the 0.003 and $0.012 \mathrm{U} / \mathrm{ml}$ dose of xanthine oxidase. Purine $(2 \mathrm{mM})$ was instilled after $15 \mathrm{~min}$ of perfusion followed by treated platelets at $19 \mathrm{~min}$, and xanthine oxidase at $20 \mathrm{~min}$.

Experiments with papavarine infusion. After instillation of purine ( $2 \mathrm{mM})$, xanthine oxidase ( 0.003 or 0.012 units $/ \mathrm{ml})$, and dehydroepiandrosterone-treated platelets as described above, papavarine $(5 \mathrm{mg}$ boluses) was instilled into the reservoir when PA pressure increased by $\geq 1 \mathrm{mmHg}$. Additional boluses of papavarine were instilled if the PA pressure did not return to baseline within $2 \mathrm{~min}$ or if the pressure increased by $\geq 1 \mathrm{mmHg}$ again later in the experiment.

Experiments with SQ 29548 infusion. Protocols using infusions of SQ 29548 were identical to those with the infusion of G-6-PD-inhibited platelets with purine and xanthine oxidase except that SQ 29548 $(100 \mu \mathrm{M})$ was instilled into the reservoir $1 \mathrm{~min}$ after purine $(16 \mathrm{~min}$ total perfusion). These experiments were performed with both concentrations of xanthine oxidase $(0.003$ and $0.012 \mathrm{U} / \mathrm{ml}$ of perfusate).

Control experiments. To exclude an effect of platelets on the isolated lung, washed platelets $\left(1.20 \pm 0.07 \times 10^{10}\right)$ without purine or xanthine oxidase were instilled into the reservoir after $19 \mathrm{~min}$ of perfusion and lungs were observed for a total duration of $60 \mathrm{~min}$. Additional experiments were performed with the instillation of platelets incubated with dehydroepiandrosterone. Dehydroepiandrosterone $\left(10^{-7} \mathrm{M}\right)$ was also added directly to the perfusate without platelets or xanthine oxidase and lungs were observed for effects on weight, lung 
lavage albumin, and PA pressure. Furthermore, dehydroepiandrosterone $\left(10^{-6} \mathrm{M}\right)$ was infused with xanthine oxidase into the perfusate without platelets to determine any combined effects of these agents.

In vitro platelet $i \mathrm{TXB}_{2}$ generation assay. Untreated washed platelets and washed platelets incubated with dehydroepiandrosterone were assayed for their ability to synthesize and release iTXB ${ }_{2}$ after stimulation with calcium ionophore A23187. Washed platelets $\left(1 \mathrm{ml}, 2 \times 10^{8}\right.$ platelets) were placed in plastic tubes in a $37^{\circ} \mathrm{C}$ shaking bath. A23187 $(1 \mu \mathrm{M})$ in DMSO was added after a 30-s stabilization period, and 20- $\mu \mathrm{l}$ samples were removed at $30,60,90,120,180$, and $300 \mathrm{~s}$. Control studies were repeated with the addition of equal volumes of the DMSO vehicle. Samples were placed into plastic tubes containing $100 \mu \mathrm{M}$ dazoxiben, a thromboxane synthetase inhibitor, in polyvinylpyrrolidine (PVP) buffer. Assays were performed on these samples for iTXB after 1:10 dilution with PVP buffer.

Similar studies were performed to test the ability of purine and xanthine oxidase in concentrations used in the isolated lung to stimulate enhanced $\mathrm{iTXB}_{2}$ release from dehydroepiandrosterone-treated platelets. Purine $(2 \mathrm{mM})$ and xanthine oxidase $(0.12 \mathrm{U} / \mathrm{ml})$ were substituted for calcium ionophore in control and dehydroepiandrosterone-treated platelets, and samples were removed and assayed for iTXB ${ }_{2}$ as described above.

Statistics. All values are expressed as mean \pm SEM. Statistical calculations were performed on a microcomputer (Macintosh SE, Apple Computer; Cupertino, CA) using a statistical package (Statview 512+, Brainpower; Calabasas, CA). One-way analysis of variance with Fisher's protected least significant difference test for multiple comparisons, Wilcoxon signed rank test, and analysis of variance with repeated measures were used as indicated. Differences were considered significant at $P$ values $<0.05$.

\section{Results}

Control experiments. Recirculating perfusate was nearly free of cellular elements. After the lungs were flushed with $500 \mathrm{ml}$ of 3\% BSA-Krebs-Henseleit solution, perfusate leukocyte counts were reduced to $4.1 \pm 2.0$ cells $/ \mathrm{cm}^{3}$, erythrocytes to $12.1 \pm 3.3$ cells $/ \mathrm{cm}^{3}$, and platelets were undetectable.

Control lungs infused with BSA-Krebs-Henseleit alone maintained stable lung weight and PA pressures during the 60-min experiments, and lung lavage albumin contents were $22.9 \pm 8.3 \mathrm{mg} / \mathrm{dl}$ after completion of perfusion (Table I). Additional control studies with the infusion of untreated washed platelets alone, dehydroepiandrosterone alone, or G-6-PD-inhibited platelets alone did not affect any measured variable (Table I). Experiments with the infusion of dehydroepiandrosterone with xanthine oxidase were similar to those with the infusion of xanthine oxidase alone (Table I).

Effects of purine and xanthine oxidase on isolated lung PA pressure and edema. Infusion of purine and xanthine oxidase $(0.003 \mathrm{U} / \mathrm{ml})(\mathrm{n}=6)$ caused increases in lung weight $(P$ $<0.05)$ (Fig. 1) and lung lavage albumin content $(P<0.05)$ (Table I) without changes in PA pressure (Fig. 1) compared with control experiments $(P=\mathrm{NS})$. Infusion of a larger xanthine oxidase dose $(0.012 \mathrm{U} / \mathrm{ml})(n=10)$ caused a greater increase in lung weight gain (Fig. 1) and lung lavage albumin content (Table II) that was associated with a rise in PA pressure (Fig. 1) above control values $(P<0.05)$.

Effects of washed platelets on purine and xanthine oxidaseinduced lung changes. Washed human platelets infused with purine and xanthine oxidase $(0.003 \mathrm{U} / \mathrm{ml})$ attenuated the oxidant-induced increases in lung weight $(P<0.05)$ (Fig. 1) and lung lavage albumin content $(P<0.05)$ (Table I) to levels that were similar to control values. Platelet infusion with the 0.003 $\mathrm{U} / \mathrm{ml}$ dose of xanthine oxidase did not alter PA pressure from baseline values (Fig. 1).

Platelets infused with the higher dose of xanthine oxidase $(0.012 \mathrm{U} / \mathrm{ml})$ decreased $(P<0.05)$ but did not completely eliminate the oxidant-induced rise in lung weight gain (Fig. 1), lung lavage albumin content (Table II), and PA perfusion pressure (Fig. 1).

Effects of G-6-PD-inhibited platelets on purine and xanthine oxidase-induced lung changes. Incubation of platelets with dehydroepiandrosterone significantly decreased $(P$ $<0.05$ ) mean platelet G-6-PD activity to $36 \%$ of control platelet values but had only a slight depressive effect on platelet glutathione concentrations $(P<0.05)$ (Table III). G-6-PD-inhibited platelets no longer attenuated the increases in lung

Table I. Effects on Isolated Perfused Rabbit Lungs of Infusion of Xanthine Oxidase $(0.003 \mathrm{U} / \mathrm{ml})$ in Experimental Conditions

\begin{tabular}{|c|c|c|c|c|}
\hline Experimental condition & $n$ & PAP & Wt gain & Albumin \\
\hline & & $m m H g$ & $g$ & $m g / d l$ \\
\hline Control-perfusate alone & 5 & $9.8 \pm 1.3$ & $-0.6 \pm 0.5$ & $22.9 \pm 8.3$ \\
\hline $\mathrm{PU}+\mathrm{XO}$ & 6 & $12.0 \pm 0.8$ & $10.6 \pm 4.7^{* \ddagger}$ & $93.0 \pm 16.6^{* \ddagger}$ \\
\hline $\mathrm{PU}+\mathrm{XO}+\mathrm{PLT}$ & 6 & $12.2 \pm 1.0$ & $1.6 \pm 0.6^{\S}$ & $46.0 \pm 8.9^{* \S}$ \\
\hline $\mathrm{PU}+\mathrm{XO}+\mathrm{DEA} / \mathrm{PLT}$ & 7 & $56.0 \pm 11.9^{* \neq \S}$ & $40.1 \pm 5.9^{* \neq \S}$ & $558.0 \pm 118.8^{* \ddagger \S}$ \\
\hline $\mathrm{PU}+\mathrm{XO}+\mathrm{DEA} / \mathrm{PLT}+\mathrm{PV}$ & 7 & $12.6 \pm 0.7$ & $15.6 \pm 6.7^{* \pm}$ & $182.6 \pm 94.9^{* \ddagger}$ \\
\hline $\mathrm{PU}+\mathrm{XO}+\mathrm{DEA} / \mathrm{ASA} / \mathrm{PLT}$ & 6 & $10.4 \pm 1.0$ & $1.0 \pm 0.6^{\S}$ & $22.9 \pm 7.0^{\S}$ \\
\hline $\mathrm{PU}+\mathrm{XO}+\mathrm{DEA} / \mathrm{PLT}+\mathrm{SQ} 29548$ & 5 & $10.2 \pm 0.7$ & $4.2 \pm 1.6^{*}$ & $14.6 \pm 5.0^{\S}$ \\
\hline PLT alone & 5 & $10.3 \pm 0.4$ & $-0.4 \pm 0.1^{\S}$ & $26.1 \pm 3.7^{\S}$ \\
\hline DEA/PLT alone & 5 & $11.0 \pm 0.9$ & $-0.5 \pm 1.1^{\S}$ & $32.7 \pm 9.7^{\S}$ \\
\hline DEA alone & 5 & $10.1 \pm 0.4$ & $-0.5 \pm 0.8^{\S}$ & $20.2 \pm 2.9^{\S}$ \\
\hline $\mathrm{DEA}+\mathrm{PU}+\mathrm{XO}$ & 3 & $11.9 \pm 0.9$ & $11.2 \pm 3.4^{\ddagger}$ & $88.2 \pm 20.0^{\ddagger}$ \\
\hline
\end{tabular}

Values are means \pm SEM. Albumin, lung lavage albumin concentration at the end of the experiment; ASA, acetylsalicylate; DEA, dehydroepiandrosterone; PAP, pulmonary artery pressure at $60 \mathrm{~min}$; PLT, platelets; PU, purine; PV, papavarine; Wt gain, increase in lung weight at 60 min; $\mathrm{XO}$, xanthine oxidase. ${ }^{*} P<0.05$ compared with respective values in control experiments; ${ }^{\ddagger} P<0.05$ compared with respective values in $\mathrm{PU}$ $+\mathrm{XO}+\mathrm{PLT}$ experiments; ${ }^{\S} P<0.05$ compared with respective values in PU + XO experiments. 

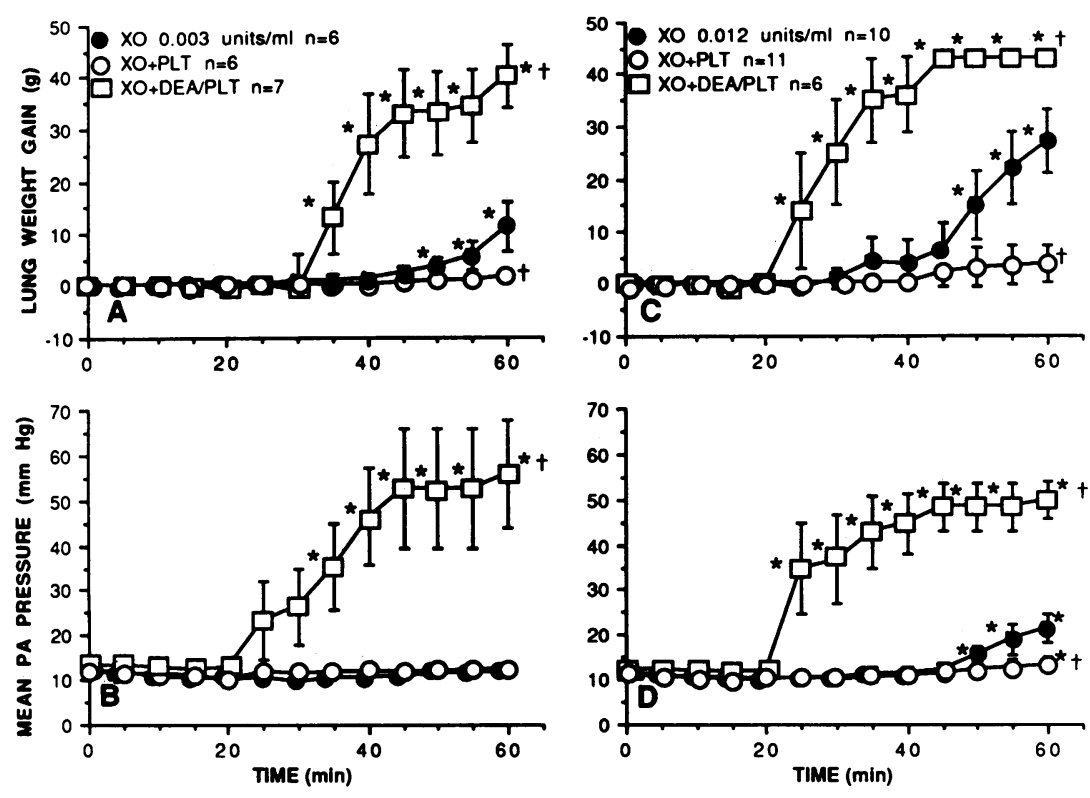

Figure 1. Time course of lung weight and mean PA pressure responses to infusion of the 0.003 ( $A$ and $B$ ) or $0.012(C$ and $D) \mathrm{U} / \mathrm{ml}$ dose of xanthine oxidase (XO) with and without either normal washed platelets (PLT) or dehydroepiandrosterone-treated platelets (DEA/PLT). ${ }^{*} P<0.05$ compared with baseline 15 -min value. $† P<0.05$ compared with the infusion of $\mathrm{XO}$ alone.

weight or lung lavage albumin concentration (Table I) that occurred after infusion of $0.003 \mathrm{U} / \mathrm{ml}$ xanthine oxidase, but rather caused a marked PA pressor response (Fig. 1) and augmention of weight gain $(n=7)$ (Fig. 1) compared with the infusion of purine and xanthine oxidase without platelets $(P$ $<0.05)$. Infusion of G-6-PD-inhibited platelets caused a similar augmentation of PA pressure, lung weight gain (Fig. 1), and lung lavage albumin concentration (Table II) when infused with the 0.012 units/ml dose of xanthine oxidase $(n=6)$.

Effects of papavarine on lung changes induced by infusion of purine, xanthine oxidase, and G-6-PD-inhibited platelets. Instillation of papavarine (range, 5-20 mg; mean, $12.5 \mathrm{mg}$ ) in experiments infused with purine, xanthine oxidase, and G-6PD-inhibited platelets prevented any rise in PA pressure at both xanthine oxidase concentrations of $0.003 \mathrm{U} / \mathrm{ml}(n=6)$ (Fig. 2) and $0.012 \mathrm{U} / \mathrm{ml}(n=6)$ (Fig. 2). Lung weights increased to levels similar to experiments infused with purine and both doses of xanthine oxidase alone, but papavarine prevented the augmentation of lung weight gain observed with G-6-PD-inhibited platelets (Fig. 2). Lung lavage albumin content was increased after infusion of papavarine with both doses of xanthine oxidase confirming the presence of lung edema (Tables I and II).
Effects of coincubation of platelets with acetylsalicylate and dehydroepiandrosterone before infusion with purine and xanthine oxidase. Platelets incubated with acetylsalicylate and dehydroepiandrosterone to inhibit both platelet cyclooxygenase and G-6-PD were infused with purine and xanthine oxidase. Infusion of treated platelets with the $0.003 \mathrm{U} / \mathrm{ml}$ concentration of xanthine oxidase $(n=6)$ did not result in the augmentation of lung edema or the pressor response observed with the infusion of G-6-PD-inhibited platelets and xanthine oxidase. Lung weight gain (Fig. 3), lung lavage albumin concentration (Table I), and PA pressure (Fig. 3) were similar compared to control experiments $(P=\mathrm{NS})$.

Infusion of G-6-PD- and cyclooxygenase-inhibited platelets with the $0.012 \mathrm{U} / \mathrm{ml}$ concentration of xanthine oxidase also did not result in augmentation of lung edema or the pressor response. Values for lung weight gain (Fig. 3) and lung lavage albumin content (Table II) were similar to the infusion of purine and xanthine oxidase $(0.012 \mathrm{U} / \mathrm{ml})$ without platelets, although the PA pressure response remained stable similar to control experiments (Fig. 3).

Effects of coinfusion of SQ 29548 with purine, xanthine oxidase, and G-6-PD-inhibited platelets. Instillation of $100 \mu \mathrm{M}$ SQ 29548 with purine, xanthine oxidase and G-6-PD-inhib-

Table II. Effects on Isolated Perfused Rabbit Lungs of Infusion of Xanthine Oxidase $(0.012 \mathrm{U} / \mathrm{ml})$ in Experimental Conditions

\begin{tabular}{|c|c|c|c|c|}
\hline Experimental condition & $n$ & PAP & Wt gain & Albumin \\
\hline & & $m m H g$ & $g$ & $m g / d l$ \\
\hline $\mathrm{PU}+\mathrm{XO}$ & 10 & $21.4 \pm 3.3^{* \ddagger}$ & $26.7 \pm 6.2^{* \ddagger}$ & $692.2 \pm 213.5^{* \ddagger}$ \\
\hline $\mathrm{PU}+\mathrm{XO}+\mathrm{PLT}$ & 11 & $13.5 \pm 1.8^{\S}$ & $3.8 \pm 3.6^{\S}$ & $42.3 \pm 16.8^{\S}$ \\
\hline $\mathrm{PU}+\mathrm{XO}+\mathrm{DEA} / \mathrm{PLT}$ & 6 & $50.0 \pm 4.1^{* \neq \S}$ & $42.8 \pm 1.6 * \pm \S$ & $750.9 \pm 202.1^{* \pm}$ \\
\hline $\mathrm{PU}+\mathrm{XO}+\mathrm{DEA} / \mathrm{PLT}+\mathrm{PV}$ & 6 & $12.7 \pm 3.5^{8}$ & $21.1 \pm 9.3^{* \ddagger}$ & $264.8 \pm 133.1^{* \neq}$ \\
\hline $\mathrm{PU}+\mathrm{XO}+\mathrm{DEA} / \mathrm{ASA} / \mathrm{PLT}$ & 6 & $24.5 \pm 5.7^{* \ddagger}$ & $21.7 \pm 11.4^{* \ddagger}$ & $407.2 \pm 176.9^{* \neq}$ \\
\hline $\mathrm{PU}+\mathrm{XO}+\mathrm{DEA} / \mathrm{PLT}+\mathrm{SQ} 29548$ & 6 & $12.8 \pm 2.1^{\S}$ & $18.3 \pm 6.7^{* \ddagger}$ & $233.5 \pm 113.9^{* *}$ \\
\hline
\end{tabular}

Values are means \pm SEM. For abbreviations, see Table I. ${ }^{*} P<0.05$ compared with respective values in control experiments; ${ }^{\ddagger} P<0.05$ compared with respective values in PU + XO + PLT experiments; ${ }^{\S} P<0.05$ compared with respective values in PU + XO experiments. 
Table III. Effects of Dehydroepiandrosterone on Platelet G-6-PD Activity and Glutathione Content

\begin{tabular}{lcc}
\hline \multicolumn{1}{c}{ Administered agent } & G-6-PD activity & GSH concentration \\
\hline & $\begin{array}{c}\text { nmol NADPH/min/ } \\
10^{9} \text { platelets }\end{array}$ & nmol GSH $/ 10^{9}$ platelets \\
Control $(n=5)$ & $95.8 \pm 13.0$ & $17.4 \pm 0.4$ \\
$10^{-6} \mathrm{M} \mathrm{DEA}(n=5)$ & $34.5 \pm 11.0^{*}$ & $15.6 \pm 0.4^{*}$ \\
\hline
\end{tabular}

Values are means \pm SEM. DEA, dehydroepiandrosterone; GSH, glutathione. ${ }^{*} P<0.05$ compared with respective values in control experiments.

ited platelets prevented the augmented lung edema and pressor response noted with xanthine oxidase and G-6-PD-inhibited platelets. Experiments with the $0.003 \mathrm{U} / \mathrm{ml}$ dose of xanthine oxidase $(n=5)$ demonstrated minimal weight gain not significantly different than' control (Fig. 4) and no increase in lung lavage albumin (Table I) or PA pressure (Fig. 4). Experiments with the $0.012 \mathrm{U} / \stackrel{\mathrm{m}}{\mathrm{m}}$ l dose of xanthine oxidase $(n=6)$ demonstrated increases in lung weight (Fig. 4) and lung lavage albumin content (Table II) similar to the infusion of purine and xanthine oxidase without platelets, but no increases in PA pressure compared with control (Fig. 4).

Perfusate levels of $i \mathrm{TXB}_{2}$. Perfusate samples in all experimental groups were assayed for iTXB2. All samples were below the minimal detectable level $(70 \mathrm{pg} / \mathrm{ml})$ for the assay (data not shown).

In vitro platelet generation of $i T X B_{2}$. Control platelets and platelets treated with dehydroepiandrosterone were assayed in vitro for $\mathrm{TXB}_{2}$ generation after stimulation by calcium ionophore A $23187(n=3)$. There were no differences between groups in rate or degree of $\mathrm{iTXB}_{2}$ production after stimulation with ionophore (Fig. 5). Unstimulated platelets had stable basal levels of $\mathrm{iTXB}_{2}$ generation.

Control or dehydroepiandrosterone-treated platelets did not release detectable iTXB ${ }_{2}$ after incubation with purine and xanthine oxidase in concentrations used in the isolated perfused lung (data not shown).

\section{Discussion}

This study indicates that normal platelets attenuate oxidantinduced lung edema and pulmonary hypertension and that this effect is mediated through platelet G-6-PD activity. Furthermore, the study demonstrates that G-6-PD-inhibited platelets augment oxidant-induced lung edema through platelet cyclooxygenase-dependent hydrostatic mechanisms. The ex vivo model allows detailed examination of the contribution of platelets to measured variables because the lung perfusate does not contain other nonplatelet cellular elements. Additionally, various enzyme inhibiting agents can be incubated with platelets in vivo and removed by washing before instillation of the platelets into the isolated lung system thereby avoiding concurrent inhibition of lung enzyme activity.

These studies complement and extend our previous investigations that identified the importance of the platelet glutathione redox cycle in the mechanisms of platelet attenuation of oxidant lung injury (19). Platelets did not prevent xanthine oxidase-induced lung injury if they were incubated with agents that either conjugated reduced glutathione, inhibited glutathione reductase, or prevented glutathione synthesis before infusion into the isolated rabbit lung. Inhibition of platelet catalase, however, did not significantly interfere with platelet protective mechanisms (19).

The present investigation used dehydroepiandrosterone as a specific inhibitor of G-6-PD $(20,21)$, a necessary enzyme for the generation of NADPH that is required for the reduction of oxidized glutathione by glutathione reductase $(22,23)$. Because complete extinction of platelet glutathione regeneration results in cytoskeletal cross-linking and cellular dysfunction (28), platelet G-6-PD activity was inhibited to only $36 \%$ of control values so as to allow sufficient enzyme activity to maintain normal glutathione content and platelet function during basal conditions. This degree of enzyme inhibition simulates the clinical disorder of G-6-PD deficiency wherein af-
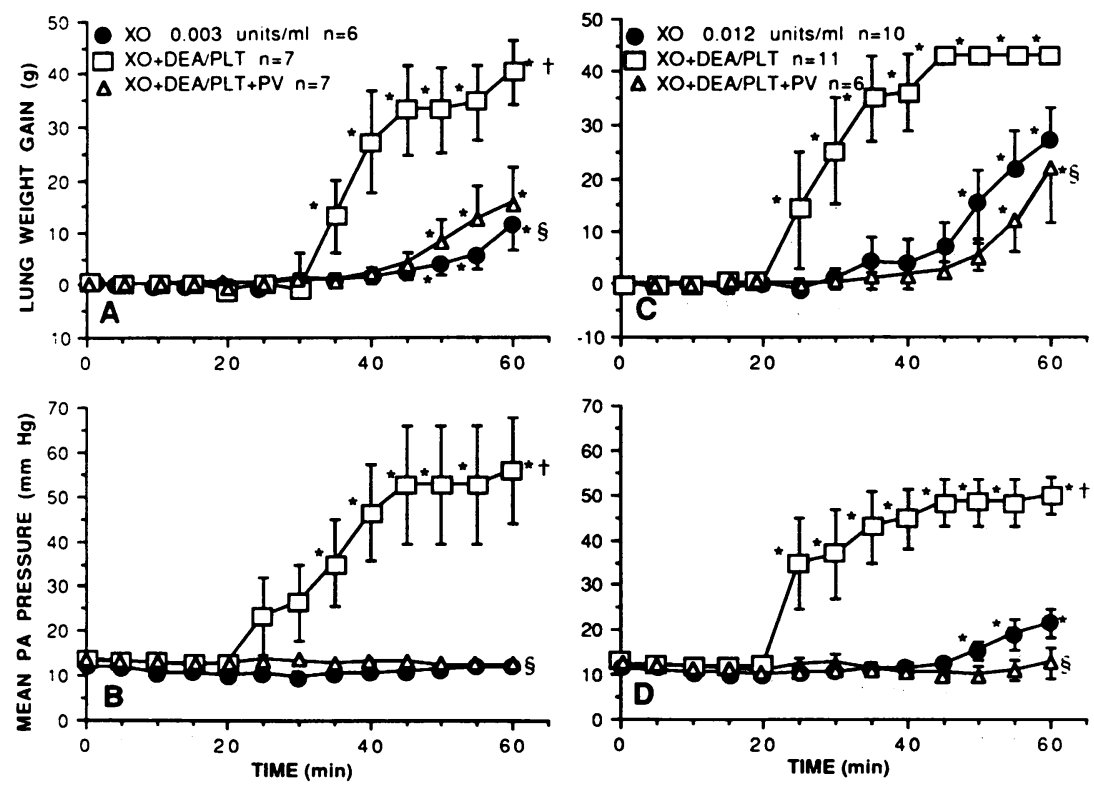

Figure 2. Time course of lung weight and mean PA pressure responses to infusion of the 0.003 ( $A$ and $B$ ) or $0.012(C$ and $D) \mathrm{U} / \mathrm{ml}$ dose of xanthine oxidase (XO) and dehydroepiandrosterone-treated platelets (DEA/PLT) with or without coinfusion of papavarine $(\mathrm{PV}) .{ }^{*} P<0.05$ compared with baseline 15 -min value. $\dagger P<0.05$ compared with the infusion of $\mathrm{XO}$ alone. $\S P<0.05$ compared with the infusion of $\mathrm{XO}$ with DEA/PLTS. 

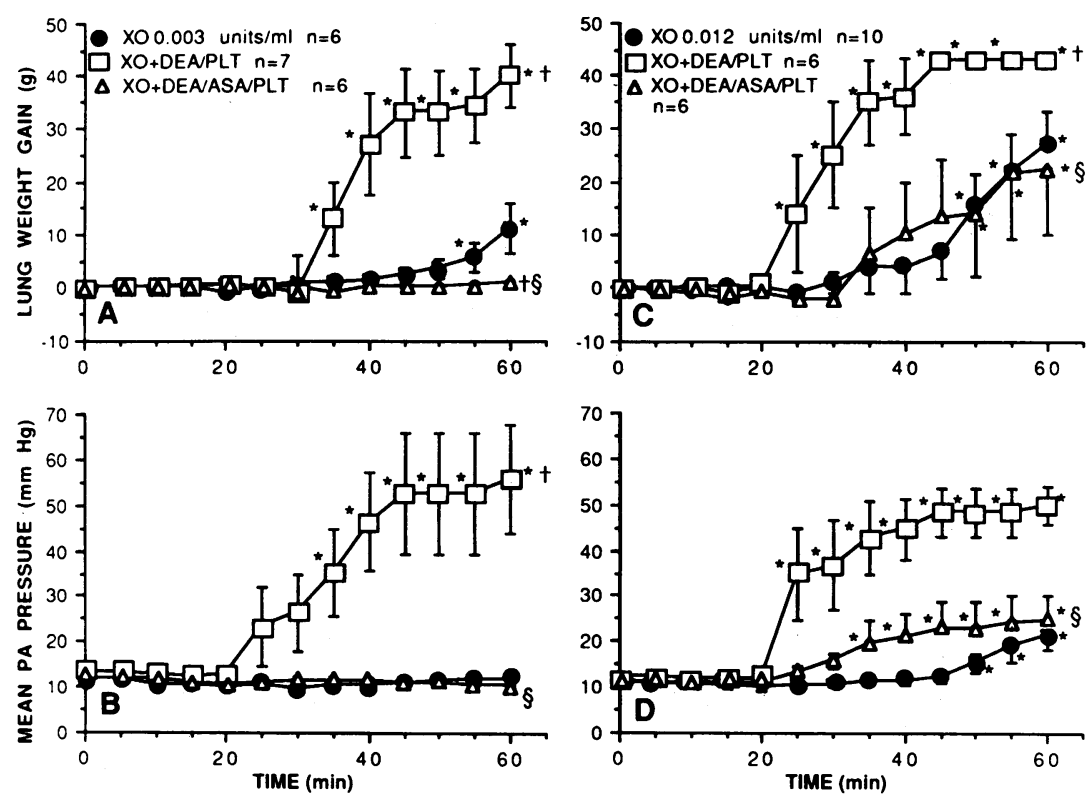

Figure 3. Time course of lung weight and mean PA pressure responses to the infusion of the 0.003 $(A$ and $B$ ) or $0.012(C$ and $D) \mathrm{U} / \mathrm{ml}$ dose of XO and dehydroepiandrosterone-treated platelets (DEA/PLT) with or without coincubation of the platelets with acetylsalicylate (ASA) $* P<0.05$ compared with baseline 15 -min value. $† P<0.05$ compared with the infusion of XO alone. $\S P$ $<0.05$ compared with the infusion of $\mathrm{XO}$ with DEA/PLTS.

fected erythrocytes function normally, except when oxidants overwhelm the regenerative capacity for reduced glutathione leading to hemolysis (29). Likewise, dehydroepiandrosteronetreated platelets in our study had a normal response to calcium ionophore but an inadequate protective effect against lung edema during xanthine oxidase-induced oxidant stress.

The observed absence of platelet attenuation of oxidant lung injury by G-6-PD-inhibited platelets is further evidence of the importance of the platelet glutathione redox cycle in protective mechanisms. These ex vivo studies combined with the in vitro observation that platelets have an avid capacity to reduce extracellular $\mathrm{H}_{2} \mathrm{O}_{2}$ (30) suggest that platelets can metabolize hydroperoxides in the pulmonary circulation, thereby limiting oxidant-induced membrane injury and resultant increased membrane permeability.

These investigations further characterize the contribution of altered hydrostatic pressure on platelet attenuation of lung edema. Infusion of the $0.012 \mathrm{U} / \mathrm{ml}$ dose of xanthine oxidase into isolated lungs, as previously reported (19), caused a rise in PA pressure in addition to increased lung weight and lung lavage albumin content; coinfusion of washed human platelets decreased both lung edema and the PA pressure rise, suggesting the possibility that one mechanism whereby platelets attenuated edema was through a reduction in capillary hydrostatic forces. Additional studies were performed using the $0.003 \mathrm{U} / \mathrm{ml}$ dose of xanthine oxidase that caused a less severe increase in lung weight and lung lavage albumin content that was associated with PA perfusion pressures similar to control values. Washed platelets prevented increased lung weight and lung lavage albumin content without affecting PA pressure, thereby demonstrating that antioxidant-intact platelets directly limit membrane permeability separate from their capacity to attenuate pulmonary vasoconstriction in this model.

Lung edema, as determined by lung weight gain, occurred
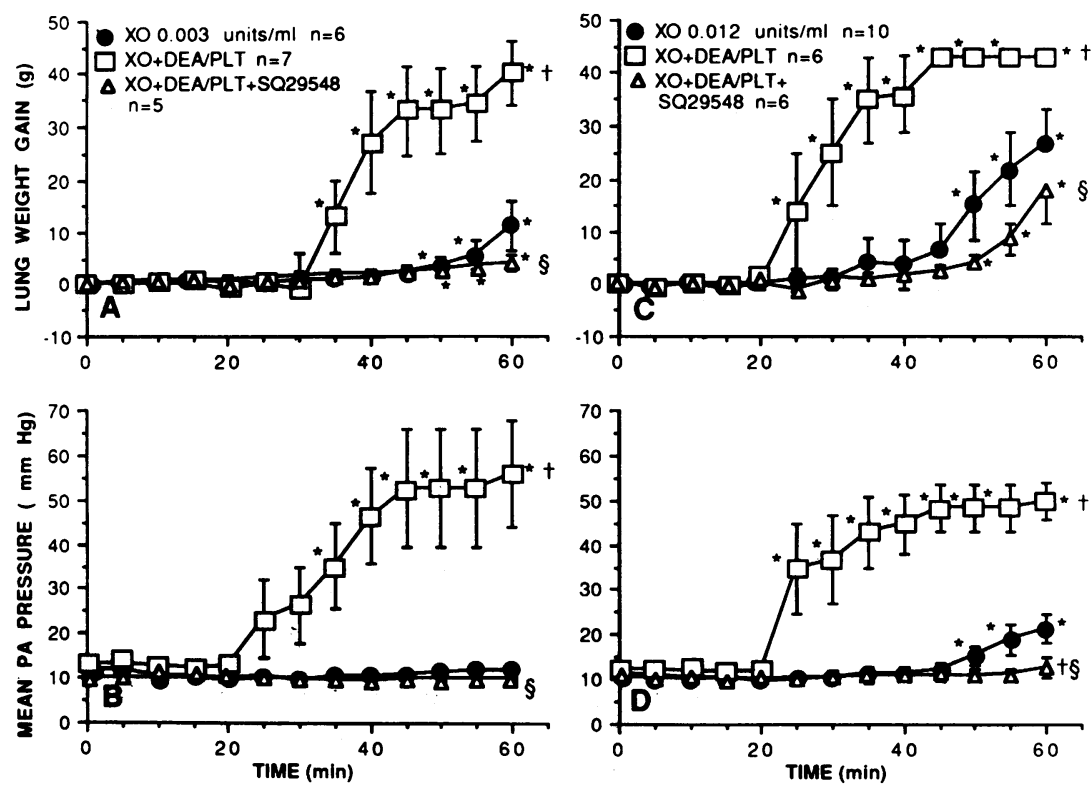

Figure 4. Time course of lung weight and mean PA pressure responses to the infusion of the 0.003 $(A$ and $B)$ or $0.012(C$ and $D) \mathrm{U} / \mathrm{ml}$ dose of XO and dehydroepiandrosterone-treated platelets (DEA/PLT) with or without coinfusion of the $\mathrm{TXA}_{2}$-prostaglandin endoperoxide receptor site antagonist (SQ 29548). ${ }^{*} P<0.05$ compared with baseline 15 -min value. $\dagger P<0.05$ compared with the infusion of $\mathrm{XO}$ alone. $\S P<0.05$ compared with the infusion of $\mathrm{XO}$ with DEA/PLTS. 


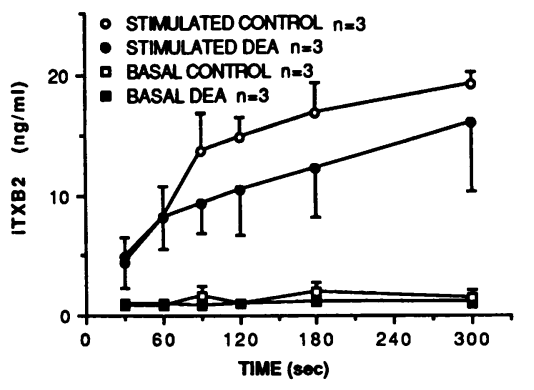

Figure 5. TXA 2 generation curve of control platelets or platelets incubated with dehydroepiandrosterone (DEA). Platelets were unstimulated or stimulated by A23187 $(1 \mu \mathrm{M})$. There was no difference between the ionophorestimulated control and DEA-treated group.

more rapidly and to a greater degree in the presence of G-6PD-inhibited platelets after infusion of either dose of xanthine oxidase $(0.003$ and $0.012 \mathrm{U} / \mathrm{ml})$. G-6-PD-inhibited platelets augmented the PA pressor response induced by the 0.012 $\mathrm{U} / \mathrm{ml}$ dose of xanthine oxidase and generated a pressor response to the $0.003 \mathrm{U} / \mathrm{ml}$ dose of xanthine oxidase, even though this concentration of enzyme did not cause pulmonary hypertension when infused in the absence of platelets. To investigate whether G-6-PD-inhibited platelets directly increased membrane permeability or augmented lung edema through hydrostatic mechanisms, PA pressures were maintained at control levels by infusing papavarine in experiments with both doses of xanthine oxidase and antioxidant-depleted platelets. Lung edema, as measured by lung weight gain, was similar in these experiments to that observed with the infusion of purine and xanthine oxidase alone without platelets. Thus, G-6-PDinhibited platelets appear to augment oxidant-induced lung edema via vasoconstriction and increased hydrostatic forces that stress the enhanced membrane permeability. Since toxic oxygen metabolites have been demonstrated to aggregate platelets in vitro (31), the vasoconstriction may be mediated through release of platelet vasoactive substances in regions of oxidant stress in the pulmonary microcirculation. We did not observe a direct cytotoxic effect of G-6-PD-inhibited platelets.

Platelets release several vasoactive substances that could produce pulmonary hypertension and lung edema by G-6PD-inhibited platelets $(13,14)$. The contribution of cyclooxygenase-dependent products, such as endoperoxides or $\mathrm{TXA}_{2}$, was explored because they are potent vasoconstrictors at the postcapillary level $(32,33)$. Also, an inverse relationship exists between platelet glutathione content and the capacity of platelets to synthesize $\operatorname{TXA}_{2}(34,35)$. Inhibition of platelet cyclooxygenase by acetylsalicylate in G-6-PD-inhibited platelets prevented the PA pressor response and augmentation of lung injury observed after the infusion of xanthine oxidase and G-6-PD-inhibited platelets with intact cyclooxygenase enzyme.

Because cyclooxygenase inhibitors interfere with platelet generation of multiple prostaglandins, the specific contribution of endoperoxides and TXA $\mathrm{TA}_{2}$ using these agents is unclear. Therefore, the effects of the specific competitive $\mathrm{TXA}_{2}$ receptor antagonist, SQ 29548 was evaluated. This compound inhibits the receptor activated by $\mathrm{TXA}_{2}$ and prostaglandin endoperoxides on vascular smooth muscle, with only weak inhibition of contractile responses to prostaglandins $F_{2 \alpha}$ and $D_{2}$ (36). Infusion of SQ 29548 prevented the marked pressor response observed with G-6-PD-inhibited platelets at both doses of xanthine oxidase, supporting a role for either endoperoxides or $\mathrm{TXA}_{2}$ in the hydrostatic augmentation of lung edema by

G-6-PD-inhibited platelets. Infusion of SQ 29548 in contrast to incubation of G-6-PD-inhibited platelets with acetylsalicylate maintained PA pressures near control values after infusion of xanthine oxidase (Fig. 3). This observation is interpreted in consideration of previous findings that isolated lungs infused with xanthine oxidase develop $\mathrm{TXA}_{2}$-induced pulmonary hypertension and the source of $\mathrm{TXA}_{2}$ is resident lung cells (37). Instillation of SQ 29548 into the reservoir would inhibit the effects of the endogenous $\mathrm{TXA}_{2}$ production; incubation of platelets with acetylsalicylate before washing and infusion into the isolated lung would inhibit only the platelet component of eicosanoid release.

A role for $\mathrm{TXA}_{2}$ could not be confirmed by assay of lung perfusate for $\mathrm{TXB}_{2}$ since these samples did not contain measureable levels of $i \mathrm{TXB}_{2}$. These findings are in contrast to previous investigations in isolated rabbit lungs where infusion of xanthine oxidase alone caused lung edema and pulmonary hypertension accompanied by a fivefold increase in perfusate $\mathrm{TXB}_{2}$ levels compared to baseline values (37). These previous studies, however, used larger doses of xanthine oxidase $(0.3$ $\mathrm{U} / \mathrm{ml}$ ). The smaller doses used in these studies may be associated with a decreased lung release of $\mathrm{TXA}_{2}$ compared with these previous investigations, thus precluding our ability to measure $\mathrm{TXB}_{2}$.

The absence of detectable iTXB ${ }_{2}$ in perfusate samples does not exclude $\mathrm{TXA}_{2}$ in the pulmonary pressor response induced by G-6-PD-inhibited platelets and xanthine oxidase. The possibility exists that platelets in the pulmonary microcirculation release $\mathrm{TXA}_{2}$ in close proximity to receptor sites, resulting in a sufficient local concentration to promote vasoconstriction. Furthermore, $\mathrm{TXB}_{2}$ is rapidly taken up by tissues after formation (38) so that circulating $\mathrm{TXB}_{2}$ may reflect only a small fraction of $\mathrm{TXA}_{2}$ production.

We attempted to indirectly identify a role for $\mathrm{TXA}_{2}$ by demonstrating its increased synthesis by G-6-PD-inhibited platelets. In contrast to previous investigations that demonstrated enhanced release of $\mathrm{TXA}_{\mathbf{2}}$ from glutathione-deficient platelets $(34,35)$, platelets treated with dehydroepiandrosterone did not have augmented synthesis of $\mathrm{TXA}_{2}$ as measured by $\mathrm{iTXB}_{2}$ production. We additionally did not demonstrate that dehydroepiandrosterone-treated platelets generated $\mathrm{iTXB}_{2}$ after stimulation with purine and xanthine oxidase in concentrations instilled in the isolated lung studies. Although previous investigators demonstrated platelet aggregation and release of serotonin after incubation with xanthine and xanthine oxidase (31), a lower concentration of xanthine oxidase was used in the present studies.

This ex vivo investigation may assist in interpreting previous conflicting whole animal studies demonstrating that platelets can either maintain normal pulmonary membrane permeability (39), augment lung injury (12), have no effect on membrane permeability $(40)$, or generate pulmonary hypertension in models of acute lung injury $(15,41)$. In models of injury that generate oxidant stress, the antioxidant capacity of the platelets may determine whether they neutralize toxic oxygen metabolites before membrane injury occurs or whether they participate in pathogenetic events by releasing vasoactive substances that augment underlying membrane permeability defects. Considering that platelets have additional potential mechanisms for participation in oxidative processes, such as release of adenine nucleotides that enhance neutrophil release of superoxide anion (42), the platelet may play an important role in regulating the degree of oxidant-induced lung injury. 


\section{Acknowledgments}

We wish to thank Dr. David Jollow, Jennifer Schulte, and Pat Germroth for assistance in the performance of these studies and Dr. Charlie Strange for helpful comments.

This work supported in part by the Medical University of South Carolina Biomedical Research Support Grant of 1986-87, and HL-29566, HL-01992, and GM-2767 from the National Institutes of Health.

\section{References}

1. Aursnes, I., and O. O. Pedersen. 1979. Petechial hemorrhage in the ciliary processes of thrombocytopenic rabbits. An electron microscopic study. Microvasc. Res. 17:12-21.

2. Gaydos, L. A., E. J. Freireich, and N. Mantel. 1962. The quantitative relation between platelet count and hemorrhage in patients with acute leukemia. N. Engl. J. Med. 266:905-909.

3. Aursnes, I. 1974. Increased permeability of capillaries to protein during thrombocytopenia: an experimental study in the rabbit. Microvasc. Res. 7:283-295.

4. Lo, S. K., K. E. Burhop, J. E. Kaplan, and A. B. Malik. 1988. Role of platelets in maintenance of pulmonary vascular permeability to protein. Am. J. Physiol. 254:H763-H771.

5. Suaudeau, J., A. Carvalho, W. G. Austen, and A. J. Erdmann. 1979. The importance of platelets for the isolated lamb heart perfused at $13^{\circ} \mathrm{C}$. Am. J. Physiol. 251:C671-C680.

6. Dodds, W. J., L. R. Raymond, and J. H. Pert. 1973. Isolated kidney perfusion: a model for testing platelet function. Proc. Soc. Exp. Biol. Med. 144:189-194.

7. Gimbone, M. A., R. H. Aster, R. S. Cotran, J. Corkery, J. H. Jandl, and J. Folkman. 1969. Preservation of vascular integrity in organs perfused in vitro with a platelet-rich medium. Nature (Lond.). 222:33-36.

8. Fantone, J. C., R. G. Kunkel, and D. A. Kinnes. 1984. Potentiation of $\alpha$-naphthyl thiourea-induced lung injury by prostaglandin $\mathrm{E}_{1}$ and platelet depletion. Lab. Invest. 50:703-710.

9. Slezak, S., D. E. Symer, and H. S. Shin. 1987. Platelet-mediated cytotoxicity. J. Exp. Med. 166:489-505.

10. Jørgensen, L., A. G. Grøthe, T. Larsen, R. L. Kinlough-Rathbone, and J. F. Mustard. 1986. Injury to cultured endothelial cells by thrombin-stimulated platelets. Lab. Invest. 54:408-415.

11. Hughes, A., and R. S. Tonks. 1962. Intravascular platelet clumping in rabbits. J. Pathol. Bacteriol. 84:379-390.

12. Tvedten, H. W., G. O. Till, and P. A. Ward. 1985. Mediators of lung injury in mice following systemic activation of complement. $\mathrm{Am}$. J. Pathol. 119:92-100.

13. Cohen, I. 1980. Platelet structure and function, role of prostaglandins. Ann. Clin. Lab. Sci. 10:187-194.

14. Holmsen, H., H. J. Day, and H. Stormorken. 1969. The blood platelet release reaction. Scand. J. Haematol. Suppl. 8:3-26.

15. Heffner, J. E., S. A. Shoemaker, E. M. Canham, M. Patel, I. F. McMurtry, H. G. Morris, and J. E. Repine. 1983. Acetyl glyceryl ether phosphorylcholine-stimulated human platelets cause pulmonary hypertension and edema in isolated rabbit lungs. J. Clin. Invest. 71:351357.

16. Pietra, G. G., J. R. Ruttner, W. Wust, and W. Glinz. 1981. The lung after trauma and shock. Fine structure of alveolar-capillary barrier in 23 autopsies. J. Trauma. 21:454-462.

17. Hill, J. D., J. L. Ratcliff, J. O. W. Parrott. 1976. Pulmonary pathology in acute respiratory insufficiency: lung biopsy as a diagnostic tool. J. Thorac. Cardiovasc. Surg. 71:64-71.

18. Heffner, J. E., S. A. Sahn, and J. E. Repine. 1987. The role of platelets in the adult respiratory distress syndrome. Culprits or bystanders? Am. Rev. Respir. Dis. 135:482-492.

19. Heffner, J. E., S. A. Katz, P. V. Halushka, and J. A. Cook. 1988. Human platelets attenuate oxidant injury in isolated rabbit lungs. $J$. Appl. Physiol. 65:1258-1266.

20. Raineri, R., and H. R. Levy. 1970. On the specificity of steroid interaction with mammary glucose 6-phosphate dehydrogenase. Biochemistry. 9:2233-2243.
21. Marks, P. A., and J. Banks. 1960. Inhibition of mammalian glucose-6-phosphate dehydrogenase by steroids. Proc. Natl. Acad. Sci. USA. 46:447-452.

22. Szeinberg, A., and P. A. Marks. 1961. Substances stimulating glucose catabolism by oxidative reactions of the pentose pathway in human erythrocytes. J. Clin. Invest. 40:914-924.

23. Jacob, H. S., and J. H. Jandl. 1966. Effects of sulfhydryl inhibition on red blood cells. II. Glutathione in the regulation of the hexose monophosphate pathway. J. Biol. Chem. 241:4243-4250.

24. Koufos, A., and A. L. Sagone. 1980. Effects of oxidant stress on the hexose monophosphate shunt pathway of platelets. Blood. 55:835-840.

25. Marks, P. A. 1966. Glucose 6-phosphate dehydrogenase: clinical aspects. Methods Enzymol. 9:131-137.

26. Tietze, F. 1969. Enzymatic method for quantitative determination of total and oxidized glutathione. Applications to mammalian blood and other tissues. Anal. Biochem. 27:502-522.

27. Wise, W. C., J. A. Cook, P. V. Halushka, and D. R. Knapp. 1980. Protective effects of thromboxane synthetase inhibitors in rats in endotoxic shock. Circ. Res. 46:854-859.

28. Bosia, A., P. Spangenberg, D. Ghigo, R. Heller, W. Lösche, G. P. Pescarmona, U. Till. 1985. Effect of GSH depletion by 1-chloro2,4-dinitrobenzene on human platelet aggregation, arachidonic acid oxidative metabolism and cytoskeletal proteins. Thromb. Res. 37:423-434.

29. Keller, D. F. 1971. G-6-PD Deficiency. CRC Press, Cleveland, OH. 17-21.

30. Holmsen, H., and L. Robkin. 1977. Hydrogen peroxide lowers ATP levels in platelets without altering adenylate energy charge and platelet functions. J. Biol. Chem. 252:1752-1757.

31. Handin, R. I., R. Karabin, G. J. Boxer. 1977. Enhancement of platelet function by superoxide anion. J. Clin. Invest. 59:959-965.

32. Bowers, R. E., E. F. Ellis, K. L. Brigham, and J. A. Oates. 1979. Effects of prostaglandin cyclic endoperoxides on the lung circulation of unanesthetized sheep. J. Clin. Invest. 63:131-137.

33. Kubo, K., and T. Kobayoshi. 1985. Effects of OKY-046, a selective thromboxane synthetase inhibitor, on endotoxin-induced lung injury in unanesthetized sheep. Am. Rev. Respir. Dis. 132:494495.

34. Guidi, G., R. Schiavon, A. Biasioli, and G. Perona. 1984. The enzyme glutathione peroxidase in arachidonic acid metabolism of human platelets. J. Lab. Clin. Med. 104:574-582.

35. Thomas, G., V. Skrinska, F. V. Lucas, and O. P. Schumacher. 1985. Platelet glutathione and thromboxane synthesis in diabetes. Diabetes. 34:951-954.

36. Ogletree, M. L., D. N. Harris, R. Greenberg, M. F. Haslanger, and M. Nakane. 1985. Pharmacological actions of SQ 29,548, a novel selective thromboxane antagonist. J. Pharmacol. Exp. Ther. 234:435441.

37. Tate, R. M., H. G. Morris, W. R. Schroeder, and J. E. Repine. 1984. Oxygen metabolites stimulate thrombexane production and vasoconstriction in isolated saline-perfused rabbit lungs. J. Clin. Invest. 74:608-613.

38. Kindahl, H. 1977. Metabolism of thromboxane $B_{2}$ in the cynomolgus monkey. Prostaglandins. 13:619-629.

39. Fantone, J. C., R. G. Kunkel, and D. A. Kinnes. 1984. Potentiation of $\alpha$-naphthyl thiourea-induced lung injury by prostaglandin $E_{1}$ and platelet depletion. Lab. Invest. 50:703-710.

40. Miles, R. G., and J. V. Hurley. 1983. The effect of thrombocytopenia on the ultrastructure and reaction to injury of vascular endothelium. Microvasc. Res. 26:273-290.

41. Hilliker, K. S., T. G. Bell, D. Lorimer, and R. A. Roth. 1984. Effects of thrombocytopenia on monocrotaline pyrrole-induced pulmonary hypertension. Am. J. Physiol. 246:H747-H753.

42. Ward, P. A., Cunningham T. W., K. K. McCulloch, S. H. Phan, J. Powell, and K. J. Johnson. 1988. Platelet enhancement of $\mathrm{O}_{2}^{-}$responses in stimulated human neutrophils. Identification of platelet factor as adenine nucleotide. Lab. Invest. 58:37-47. 